\title{
AN ANALYTICAL SOLUTION OF THE GENERALIZED EQUATION OF ENERGY TRANSPORT IN ONE-DIMENSIONAL SEMI-INFINITE DOMAINS
}

\author{
VLADIMIR V. KULISH
}

Received 23 March 2004 and in revised form 28 April 2004

This paper presents an integral solution of the generalized one-dimensional equation of energy transport with the convective term. The solution of the problem has been achieved by the use of a novel technique that involves generalized derivatives (in particular, derivatives of noninteger orders). Confluent hypergeometric functions, known as Whittaker's functions, appear in the course of the solution procedure upon applying the Laplace transform to the original transport equation. The analytical solution of the problem is written in the integral form and provides a relationship between the local values of the transported property (e.g., temperature, mass, momentum, etc.) and its flux. The solution is valid everywhere within the domain, including the domain boundary.

\section{Introduction}

This paper presents a further development of the method that was first discussed by Oldham and Spanier [12]. The same method was successfully used in numerous applications $[4,5,6]$. In recent works, the method was extended and applied to problems involving combustion [7], hyperbolic heat transfer [10,11], turbulent flows [2], and such problems in biomedical engineering as modeling of the neural response to an external stimulus [9] and the alveolar gas exchange [3].

This work is an attempt to generalize the above-mentioned method.

\section{Generalized equation of energy transport}

A generalized energy transport equation with the convective term can be obtained from the conservation equation written in the differential form

$$
\frac{\partial F(\mathbf{r}, t)}{\partial t}+\nabla \cdot \varphi(\mathbf{r}, t)=S(\mathbf{r}, t)
$$

where $F$ represents the transported property (such as, temperature, mass concentration, momentum, etc.), $\varphi$ denotes the flux of $F, S(\mathbf{r}, t)$ is the source function, whereas $\mathbf{r}$ and $t$ are the spatial and time-independent variables, respectively. 
The conservation equation involves two unknown variables, $F$ and $\varphi$, and hence must be coupled with a constitutive equation that would relate these unknown quantities.

In general, constitutive equations are but assumptions and, unlike the conservation equation, cannot be derived from fundamental principles. Therefore, the final form of the equation of energy transport depends on the form of the constitutive equation used.

In the present model, the following constitutive equation is used:

$$
\varphi(\mathbf{r}, t+\tau)=-D(\mathbf{r}, t) \nabla F(\mathbf{r}, t)+\mathbf{u}(\mathbf{r}, t) F(\mathbf{r}, t),
$$

where $D$ is the diffusion coefficient always measured in $\mathrm{m}^{2} / \mathrm{s}$ (it can be, e.g., mass diffusivity, kinematic viscosity, or the coefficient $h / m$ that appears in the Schrödinger equation, where $h$ is Planck's constant and $m$ is the particle's mass) and $\mathbf{u}(\mathbf{r}, t)$ denotes the velocity vector - therefore, quantity $F$ is transported by both diffusion (the first term in the right-hand side of the constitutive equation) and convection (the second term in the right-hand side). The parameter $\tau$ represents the time lag between the onset of the gradient of the transported quantity and the occurrence of the flux of that quantity. Hence, unlike Fick's or Fourier's constitutive equations, (2.2) accounts for a finite speed of the transport process and is more general than those equations.

The left- and right-hand sides of the constitutive relation are written for two different time moments. In order to overcome this difficulty, the left-hand side of (2.2) is expanded into the Taylor series. The constitutive equation becomes

$$
\sum_{n=0}^{\infty} \frac{\tau^{n}}{n !} \frac{\partial^{n} \varphi(\mathbf{r}, t)}{\partial t^{n}}=-D(\mathbf{r}, t) \nabla F(\mathbf{r}, t)+\mathbf{u}(\mathbf{r}, t) F(\mathbf{r}, t),
$$

where $\partial^{0} \boldsymbol{\varphi}(\mathbf{r}, t) / \partial t^{0}=\boldsymbol{\varphi}(\mathbf{r}, t)$.

Upon applying the divergence operator to both parts of (2.3), the latter becomes

$$
\sum_{n=0}^{\infty} \frac{\tau^{n}}{n !} \frac{\partial^{n}[\nabla \cdot \varphi(\mathbf{r}, t)]}{\partial t^{n}}=-\nabla \cdot[D(\mathbf{r}, t) \nabla F(\mathbf{r}, t)]+\nabla \cdot[\mathbf{u}(\mathbf{r}, t) F(\mathbf{r}, t)] .
$$

Now it follows from $(2.1)$ that $\nabla \cdot \varphi(\mathbf{r}, t)=-\partial F(\mathbf{r}, t) / \partial t+S(\mathbf{r}, t)$. Upon substituting this into (2.4) and rearranging the terms, the equation becomes

$$
\begin{aligned}
\sum_{n=0}^{\infty} \frac{\tau^{n}}{n !} & \frac{\partial^{n+1} F(\mathbf{r}, t)}{\partial t^{n+1}}+\nabla \cdot[\mathbf{u}(\mathbf{r}, t) F(\mathbf{r}, t)] \\
& =\nabla \cdot[D(\mathbf{r}, t) \nabla F(\mathbf{r}, t)]+S(\mathbf{r}, t)+\sum_{n=1}^{\infty} \frac{\tau^{n}}{n !} \frac{\partial^{n} S(\mathbf{r}, t)}{\partial t^{n}}
\end{aligned}
$$

Equation (2.5) is the generalized equation of energy transport. It reduces to the classical diffusion (heat) equation if $\tau=0$ and $\mathbf{u}(\mathbf{r}, t)=0$. If $\mathbf{u}(\mathbf{r}, t)=0$ and $\tau \ll 1$ (all the terms whose order is larger than one can be neglected in the series), the generalized equation of energy transport reduces to the classical wave equation, whereas $c=\sqrt{D / \tau}$ is the propagation speed of waves (e.g., speed of light or sound). Note the presence of the apparent energy source $\sum_{n=1}^{\infty}\left(\tau^{n} / n !\right)\left(\partial^{n} S(\mathbf{r}, t) / \partial t^{n}\right)$ in $(2.5)$; it appears due to the finite time lag between the excitation and the response to it. 
If the diffusion coefficient and velocity are both constant, that is, $D(\mathbf{r}, t)=D$ and $\mathbf{u}(\mathbf{r}, t)=u$, (2.5) becomes

$$
\sum_{n=0}^{\infty} \frac{\tau^{n}}{n !} \frac{\partial^{n+1} F(\mathbf{r}, t)}{\partial t^{n+1}}+u \nabla F(\mathbf{r}, t)=D \nabla^{2} F(\mathbf{r}, t)+S(\mathbf{r}, t)+\sum_{n=1}^{\infty} \frac{\tau^{n}}{n !} \frac{\partial^{n} S(\mathbf{r}, t)}{\partial t^{n}} .
$$

\section{Problem formulation}

Consider an energy transport process that occurs in a homogeneous (no preferred direction), semi-infinite domain whose boundary moves with a constant speed $u$ and whose diffusion coefficient depends neither on spatial variable nor on time. In this case, the generalized equation of energy transport becomes

$$
\begin{aligned}
& \sum_{n=0}^{\infty} \frac{\tau^{n}}{n !} \frac{\partial^{n+1} F(r, t)}{\partial t^{n+1}}+u \frac{\partial F(r, t)}{\partial r} \\
& \quad=D\left[\frac{\partial^{2} F(r, t)}{\partial r^{2}}+\frac{2 \gamma}{r} \frac{\partial F(r, t)}{\partial r}\right]+S(r, t)+\sum_{n=1}^{\infty} \frac{\tau^{n}}{n !} \frac{\partial^{n} S(r, t)}{\partial t^{n}} .
\end{aligned}
$$

The parameter $\gamma$ characterizes the domain geometry. Thus, $\gamma=0$ corresponds to the domain with the flat boundary (no curvature); $\gamma= \pm 1$ represents the spherical case with the convex and the concave boundary, respectively; whereas $\gamma= \pm 1 / 2$ describes the cylinder whose boundary is either convex $(\gamma=1 / 2)$ or concave $(\gamma=-1 / 2)$.

The spatial variable $r=x \pm R$, where $x$ is the actual distance from the origin and $R$ is the initial radius of curvature. Note that the sign of $R$ must be the same as the sign of $\gamma$.

Initially, at $t=0$, the domain is in equilibrium with a constant value of the transported quantity, $F_{0}$, throughout the domain, $0 \leq r<+\infty$.

As the energy transport process goes on, the condition $\lim _{r \rightarrow \infty} F(r, t)=F_{0}$ must be imposed in order to comply with the principle of energy conservation.

At this point, the second boundary condition is deliberately not imposed. This issue will be clarified in the following section.

\section{Solution procedure}

Upon introducing the new variable $\rho=r / \sqrt{D}$ and the excess of the transported quantity $\hat{F}=F-F_{0}$, the transport equation becomes

$$
\sum_{n=0}^{\infty} \frac{\tau^{n}}{n !} \frac{\partial^{n+1} \hat{F}(\rho, t)}{\partial t^{n+1}}=\frac{\partial^{2} \hat{F}(\rho, t)}{\partial \rho^{2}}+2\left(\frac{\gamma}{\rho}-\omega\right) \frac{\partial \hat{F}(\rho, t)}{\partial \rho}+\sum_{n=0}^{\infty} \frac{\tau^{n}}{n !} \frac{\partial^{n} S(\rho, t)}{\partial t^{n}},
$$

where $\omega=u / 2 \sqrt{D}$ and $\partial^{0} S(\rho, t) / \partial t^{0}=S(\rho, t)$.

The initial condition becomes $\hat{F}(\rho, 0)=0$ and the boundary condition is now $\lim _{\rho \rightarrow \infty} \hat{F}(\rho, t)=0$.

Upon taking the Laplace transform of (4.1), the latter becomes

$$
\frac{d^{2} \Phi(\rho ; s)}{d \rho^{2}}+2\left(\frac{\gamma}{\rho}-\omega\right) \frac{d \Phi(\rho ; s)}{d \rho}-s e^{s \tau} \Phi(\rho ; s)=-e^{s \tau} Q(\rho, s),
$$


where $\Phi$ is the Laplace transform of the excess of the transported quantity $\hat{F}, s$ is the Laplace transform variable, and $Q(\rho, s)$ represents the Laplace transform of the source function $S$, provided that this Laplace transform exists.

The general solution of (4.2) is

$$
\Phi(\rho ; s)=\left\{C_{1}(s) M_{\kappa, \mu}[2 \rho f(s)]+C_{2}(s) W_{\kappa, \mu}[2 \rho f(s)]\right\} \frac{e^{\omega \rho}}{\rho^{\gamma}}+P(\rho, s),
$$

where $f(s)=\sqrt{s e^{s \tau}+\omega^{2}}, P(\rho, s)$ is a particular solution of (4.2), $C_{1}(s)$ and $C_{2}(s)$ are two arbitrary functions of the Laplace transform variable $s, M_{\kappa, \mu}(z)$ and $W_{\kappa, \mu}(z)$ are Whittaker's functions defined as

$$
\begin{aligned}
& M_{\kappa, \mu}(z)=e^{-z / 2} z^{\mu+1 / 2} M\left(\frac{1}{2}+\mu-\kappa, 1+2 \mu, z\right), \\
& W_{\kappa, \mu}(z)=e^{-z / 2} z^{\mu+1 / 2} U\left(\frac{1}{2}+\mu-\kappa, 1+2 \mu, z\right),
\end{aligned}
$$

where $z=2 \rho f(s), \kappa=\gamma \omega / f(s)$, and $\mu=\gamma-1 / 2$. Note that the value of $z$ increases as $\rho$ increases. Functions $M$ and $U$ in (4.4) are Kummer's confluent hypergeometric functions defined as

$$
\begin{gathered}
U(a, b, z)=\frac{\pi}{\sin (\pi b)}\left[\frac{M(a, b, z)}{\Gamma(1+a-b) \Gamma(b)}-z^{1-b} \frac{M(1+a-b, 2-b, z)}{\Gamma(a) \Gamma(2-b)}\right] \\
M(a, b, z)=1+\frac{a z}{b}+\frac{(a)_{2} z^{2}}{(b)_{2} 2 !}+\cdots+\frac{(a)_{n} z^{n}}{(b)_{n} n !}+\cdots,
\end{gathered}
$$

where $(a)_{n}=a(a+1)(a+2) \cdots(a+n-1),(b)_{n}=b(b+1)(b+2) \cdots(b+n-1)$, and $(a)_{0}=(b)_{0}=1$ (see $[1$, page 504$\left.]\right)$.

Written in terms of Kummer's functions, (4.3) becomes

$$
\Phi(z ; s)=\left[C_{1}(s) M(a, b, z)+C_{2}(s) U(a, b, z)\right][2 f(s)]^{\gamma} e^{-z(1+\omega / f(s)) / 2}+P(z, s),
$$

where $a=\gamma(1-\omega / f(s))$ and $b=2 \gamma$.

Now, since $\lim _{|z| \rightarrow \infty} M(a, b, z)=(\Gamma(b) / \Gamma(a)) e^{z} z^{a-b}$ (see [1, page 504]), the first term in (4.6) becomes unbounded for large values of $z$. This, however, contradicts the boundary condition $\lim _{z \rightarrow \infty} \Phi(z ; s)=0$. Hence, for the solution to be bounded, the arbitrary function $C_{1}(s)$ must be identically zero. On the other hand, $\lim _{|z| \rightarrow \infty} U(a, b, z)=z^{-a}$ (see [1, page 504]). Therefore, provided that $s>0$ (this is really the case because the Laplace transform variable corresponds to time and is always positive), the second term in (4.6) decreases as $z$ increases and vanishes as $z$ becomes infinitely large. Consequently, the solution becomes

$$
\Phi(\rho ; s)=C(s) U[a, b, 2 \rho f(s)][2 f(s)]^{\gamma} e^{\rho[\omega-f(s)]}+P(\rho, s) .
$$


Upon differentiating (4.7) with respect to $\rho$, the equation becomes

$$
\begin{aligned}
\frac{d \Xi}{d \rho}=C(s)[2 f(s)]^{\gamma}\{ & -2 a f(s) U[a+1, b+1,2 \rho f(s)] \\
& +U[a, b, 2 \rho f(s)][\omega-f(s)]\} e^{\rho[\omega-f(s)]},
\end{aligned}
$$

where $\Xi(\rho ; s)=\Phi(\rho ; s)-P(\rho ; s)$. Note that

$$
\frac{d U[a, b, 2 \rho f(s)]}{d \rho}=-2 a f(s) U[a+1, b+1,2 \rho f(s)] .
$$

Furthermore, it follows from (4.7) that

$$
C(s)=\frac{1}{U[a, b, 2 \rho f(s)]} \Xi(\rho ; s)[2 f(s)]^{-\gamma} e^{-\rho[\omega-f(s)]} .
$$

Upon substituting this into (4.8), the latter reduces to

$$
\frac{d \Xi}{d \rho}=\left\{[\omega-f(s)]+2 \gamma[\omega-f(s)] \frac{U[a+1, b+1,2 \rho f(s)]}{U[a, b, 2 \rho f(s)]}\right\} \Xi(\rho ; s) .
$$

Dividing (4.11) by $f(s)$,

$$
\begin{aligned}
-\frac{1}{f(s)} & \frac{d \Xi}{d \rho} \\
\quad & \left\{1-\frac{\omega}{f(s)}+2 \gamma\left[1-\frac{\omega}{f(s)}\right] \frac{U[\gamma(1-\omega / f(s))+1,2 \gamma+1,2 \rho f(s)]}{U[\gamma(1-\omega / f(s)), 2 \gamma, 2 \rho f(s)]}\right\} \Xi(\rho ; s) .
\end{aligned}
$$

Note the minus sign in the left-hand side.

Now, upon noticing that $U(a+1, b+1, z) / U(a, b, z)=1 / z=1 /[2 \rho f(s)]$, (4.12) simplifies to

$$
-\frac{1}{f(s)} \frac{d \Xi}{d \rho}=\left[1+\left(\frac{\gamma}{\rho}-\omega\right) \frac{1}{f(s)}-\frac{\gamma \omega}{\rho} \frac{1}{f^{2}(s)}\right] \Xi(\rho ; s) .
$$

In most practical applications, the values of the time lag $\tau$ are very small. In fact, $\tau=$ $D / c^{2}$, where $c$ is the speed of the energy-transporting waves (it is usually very large, while the diffusion coefficient $D$ is small). Hence, in this case, $f(s)=\sqrt{s e^{s \tau}+\omega^{2}}$ can be written as $f(s)=\sqrt{s(s \tau+1)+\omega^{2}}$. The inverse Laplace transform of $1 / f(s)$ is [1, page 1025]

$$
\begin{aligned}
L^{-1}\left[\frac{1}{\sqrt{s(s \tau+1)+\omega^{2}}}\right] & =\frac{1}{\sqrt{\tau}} L^{-1}\left[\frac{1}{\sqrt{(s+1 / 2 \tau)^{2}-\left(1 / 4 \tau^{2}-\omega^{2} / \tau\right)}}\right] \\
& =\frac{e^{-t /(2 \tau)}}{\sqrt{\tau}} I_{0}\left(\frac{t}{2 \tau} \sqrt{1-4 \tau \omega^{2}}\right)
\end{aligned}
$$


where $I_{0}$ is the modified Bessel function. The inverse Laplace transform of $1 / f^{2}(s)$ is [1, page 1022]

$$
\begin{aligned}
L^{-1}\left[\frac{1}{s(s \tau+1)+\omega^{2}}\right] & =\frac{1}{\tau} L^{-1}\left[\frac{1}{(s+1 / 2 \tau)^{2}-\left(1 / 4 \tau^{2}-\omega^{2} / \tau\right)}\right] \\
& =\frac{2 e^{-t /(2 \tau)}}{\sqrt{1-4 \tau \omega^{2}}} \sinh \left(\frac{t}{2 \tau} \sqrt{1-4 \tau \omega^{2}}\right) .
\end{aligned}
$$

Upon taking the inverse Laplace transform of (4.13) and restoring the original variables, the solution becomes

$$
\begin{aligned}
F(r, t)= & F_{0}-c \int_{0}^{t} e^{-(t-\zeta) /(2 \tau)} I_{0}\left(\frac{t-\zeta}{2 \tau} \sqrt{1-\frac{u^{2}}{c^{2}}}\right) \frac{\partial[F(r, \zeta)-p(r, \zeta)]}{\partial r} d \zeta \\
& +\left(\frac{u}{2 \tau c}-\frac{\gamma c}{r}\right) \int_{0}^{t} e^{-(t-\zeta) /(2 \tau)} I_{0}\left(\frac{t-\zeta}{2 \tau} \sqrt{1-\frac{u^{2}}{c^{2}}}\right)\left[F(r, \zeta)-F_{0}-p(r, \zeta)\right] d \zeta \\
& +\frac{\gamma u}{r \sqrt{1-u^{2} / c^{2}}} \int_{0}^{t} e^{-(t-\zeta) /(2 \tau)} \sinh \left(\frac{t-\zeta}{2 \tau} \sqrt{1-\frac{u^{2}}{c^{2}}}\right)\left[F(r, \zeta)-F_{0}-p(r, \zeta)\right] d \zeta \\
& +p(r, t),
\end{aligned}
$$

where $p(r, t)$ is the inverse Laplace transform of the particular solution $P(r ; s)$.

Finally, upon substituting the constitutive equation relating the transported quantity $F$ with its flux $\phi$, namely, $-\partial F(r, t) / \partial r=(1 / D)[\phi(r, t)+\tau(\partial \phi(r, t) / \partial t)-u F(r, t)]$, the solution becomes

$$
\begin{aligned}
F(x, t)= & F_{0}+\frac{1}{\ell} \int_{0}^{t} e^{-(t-\zeta) /(2 \tau)} I_{0}\left(\frac{t-\zeta}{2 \tau} \sqrt{1-\frac{u^{2}}{c^{2}}}\right)\left[\phi(x, t)+\tau \frac{\partial \phi(x, \zeta)}{\partial t}-u F(x, \zeta)+\beta(x, \zeta)\right] d \zeta \\
& +\left(\frac{u}{2 \ell}-\frac{\gamma c}{x \pm R}\right) \int_{0}^{t} e^{-(t-\zeta) /(2 \tau)} I_{0}\left(\frac{t-\zeta}{2 \tau} \sqrt{1-\frac{u^{2}}{c^{2}}}\right)\left[F(x, \zeta)-F_{0}-p(x, \zeta)\right] d \zeta \\
& +\frac{\gamma u}{(x \pm R) \sqrt{1-u^{2} / c^{2}}} \int_{0}^{t} e^{-(t-\zeta) /(2 \tau)} \sinh \left(\frac{t-\zeta}{2 \tau} \sqrt{1-\frac{u^{2}}{c^{2}}}\right)\left[F(x, \zeta)-F_{0}-p(x, \zeta)\right] d \zeta \\
& +p(x, t),
\end{aligned}
$$

where $\beta(x, t)=-D \partial p(x, t) / \partial x$ denotes the effective flux due to the presence of the source function in the original equation, and $R$ represents the radius of curvature of the surface. The sign of $R$ must be the same as the sign of the parameter $\gamma$. The parameter $\ell=c \tau$ is the "space quantum."

Equation (4.17) is an integral solution of the generalized problem of energy transport modeled by (3.1) in the case of small values of the phase lag $\tau$. It provides a relationship between the local values of the transported quantity $F$ and its flux $\phi$ and is valid everywhere within the domain, including the domain boundary. 


\section{Some special cases of the solution}

In some special cases, the solution given by (4.17) must reduce to some known solutions of the transport problem. In this section, it is shown that this is really the case.

Note that $\lim _{z \rightarrow \infty} I_{0}(z)=e^{z} / \sqrt{2 \pi z}[1$, page 377].

Hence, in the case of $\tau=0$ and $u=0,(4.17)$ reduces to

$$
\begin{aligned}
F(x, t)= & F_{0}+\frac{\partial^{-1 / 2}[\phi(x, t)-\beta(x, t)]}{\partial t^{-1 / 2}} \\
& +p(x, t)+\frac{\gamma}{x \pm R} \sqrt{D} \frac{\partial^{-1 / 2}\left[p(x, t)-F(x, t)+F_{0}\right]}{\partial t^{-1 / 2}},
\end{aligned}
$$

where the derivatives of a negative fractional order $\alpha$ are defined as

$$
\frac{d^{\alpha} f(t)}{d t^{\alpha}}=\frac{1}{\Gamma(-\alpha)} \int_{0}^{t} \frac{f(\tau) d \tau}{\sqrt{t-\tau}}
$$

for any $f(t)$ for which the above integral exists.

The solution given by (5.1) has been reported in the case of no source function in [12].

Furthermore, if $\gamma=0$ in (5.1)—which corresponds to the planar geometry-the solution becomes identical to the solution reported in [6]. If the source function is absent in the original equation, then (5.1) with $\gamma=0$ becomes the case reported in $[4,5]$.

On the other hand, if $\gamma=0, u \neq 0$, and no source function is present in (4.17), the solution coincides with the integral equation reported in [7].

Finally, in the case of $u=0$, the solution given by (4.17) becomes

$$
\begin{aligned}
F(x, t)= & F_{0}+\frac{1}{\ell} \int_{0}^{t} e^{-(t-\zeta) /(2 \tau)} I_{0}\left(\frac{t-\zeta}{2 \tau}\right)\left[\phi(x, t)+\tau \frac{\partial \phi(x, \zeta)}{\partial t}+\beta(x, \zeta)\right] d \zeta \\
& -\frac{\gamma c}{x \pm R} \int_{0}^{t} e^{-(t-\zeta) /(2 \tau)} I_{0}\left(\frac{t-\zeta}{2 \tau}\right)\left[F(x, \zeta)-F_{0}-p(x, \zeta)\right] d \zeta+p(x, t)
\end{aligned}
$$

Note that, in the case of $\gamma=0$ and no source function, the solution is identical to the integral equation reported in [8].

\section{Model validation}

To validate the model, (4.17) has been numerically solved for various sets of parameters. The physical properties of the domain were set as follows: $c=10^{3} \mathrm{~m} / \mathrm{s}$ and $D=10^{-5} \mathrm{~m}^{2} / \mathrm{s}$. Such a choice was made in order to be consistent with the results obtained in [8].

Figure 6.1 shows the normalized value of the transported quantity, $F / F_{\max }$, on the moving planar boundary for different values of the boundary speed, when the flux is constant $\left(\phi=100\right.$ units $\left./ \mathrm{m}^{2}\right)$. The solution for the latter case is well known, $F / F_{\max } \sim \sqrt{t}$. One can see from Figure 6.1 that the stronger the convective effect is the slower the solution grows.

Figure 6.2 presents the case of an expanding sphere $\left(R=4 \times 10^{-8} \mathrm{~m}\right)$. The evolution of the normalized transported property is shown on the boundary of the sphere, provided 


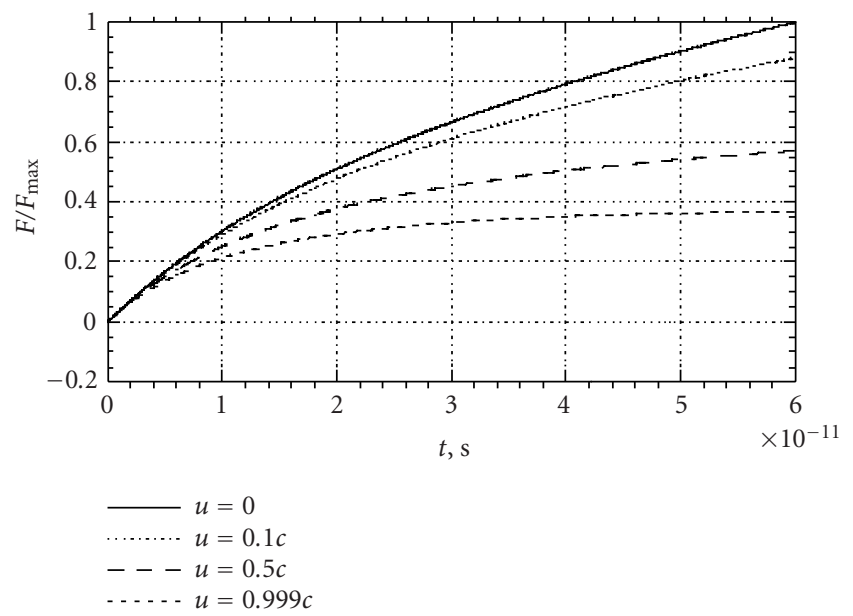

Figure 6.1. Normalized value of the transported quantity in the case of a semi-infinite domain with the moving boundary (constant flux).

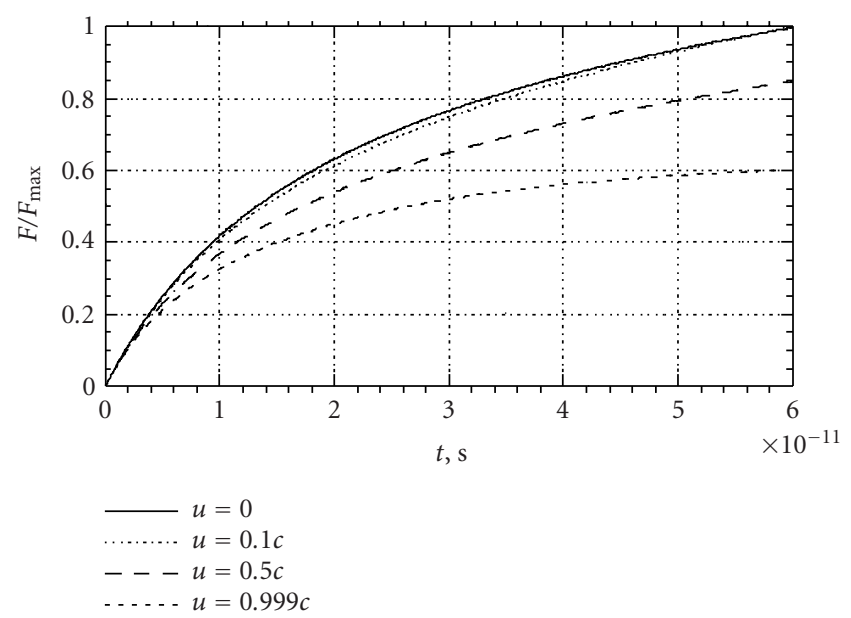

Figure 6.2. Normalized value of the transported quantity in the case of an expanding sphere (constant flux).

that the surface flux is constant $\left(\phi=100\right.$ units $\left./ \mathrm{m}^{2}\right)$. The parameters $c$ and $D$ in the transport equation were set the same as in the previous case. The solution is further compared with the case of a stationary sphere $(u=0)$.

Figure 6.3 shows the evolution of the normalized transported quantity on the moving boundary $(u=0.5 c)$ in the case of a Gaussian flux. The flux was modeled as the surface flux $\phi_{\text {surf }}(t)=\exp \left[-((t-b) / \sigma)^{2}\right]$, with $b=10$ picoseconds and $\sigma=5$ picoseconds, that mimics an almost instantaneous source of energy. Such a choice was made in order to provide a comparison with the results obtained previously for the laser pulse heating [8]. 


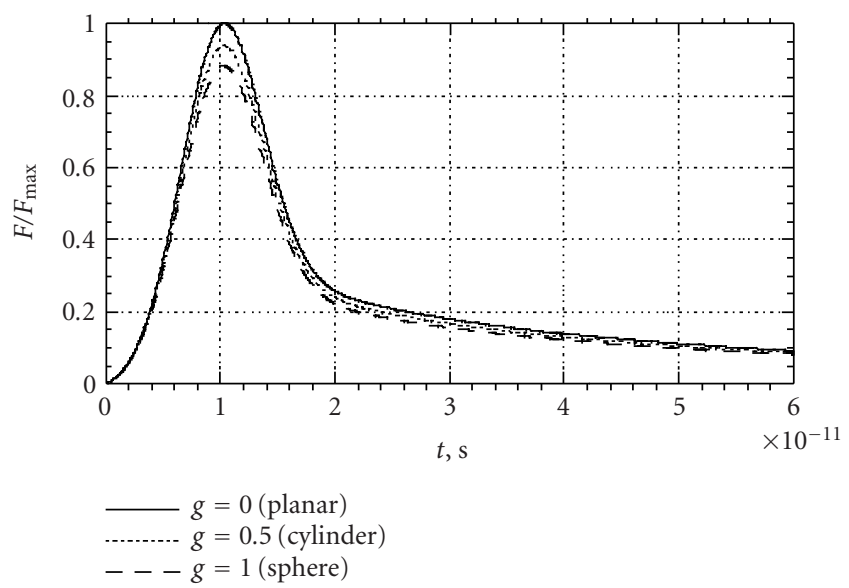

Figure 6.3. Normalized value of the transported quantity in the case of a Gaussian flux.

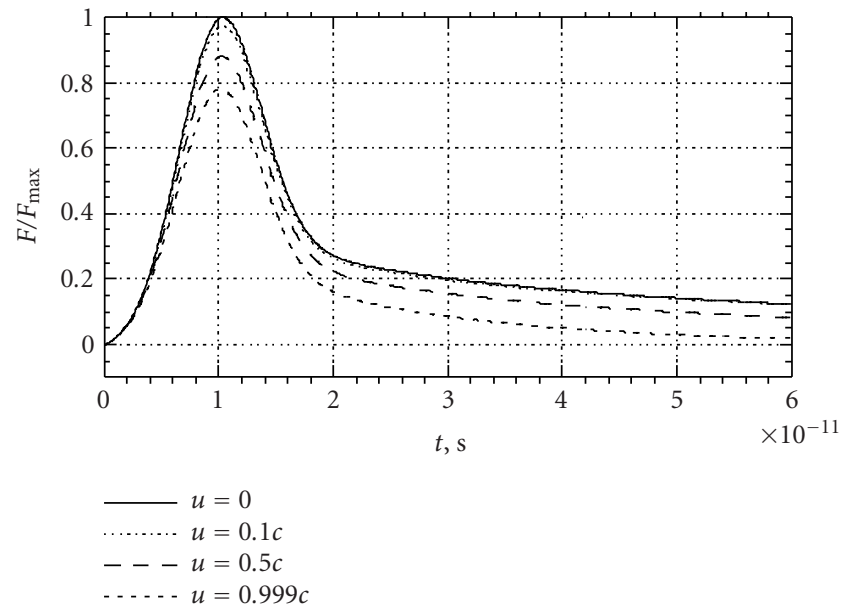

Figure 6.4. Normalized value of the transported quantity in the case of an expanding sphere (Gaussian flux).

It is evident from Figure 6.3 that for the given set of conditions, the fastest energy transport takes place in the case of the spherical geometry. Yet all the solutions converge to each other as time increases. This is easily explained from the fact that the radius of the surface curvature increases as time goes on, $\lim _{t \rightarrow \infty} R=\infty$, which corresponds to the planar geometry.

Finally, Figure 6.4 shows the results obtained in the case of an expanding sphere $(R=$ $4 \times 10^{-8} \mathrm{~m}$ ) with the Gaussian surface flux (the same as in the preceding case). The effect of convection is clearly seen in the figure. 


\section{Conclusions}

The paper presents a further development and generalization of the method that allows obtaining analytical solutions of various energy transport problems. The method is based on a technique that involves the use of generalized derivatives (sometimes reducible to derivatives of noninteger orders, known as fractional differ-integrals).

In this paper, the generalized equation of energy transport has been derived by coupling the conservation equation with the constitutive equation that represented the flux as the superposition of the classical Fick's (Fourier's) term and convective term. The constitutive equation also accounted for a possible finite time lag between the onset of the gradient of the transported quantity and its flux. The equation of energy transport thus obtained was then solved in its one-dimensional form within a semi-infinite domain whose geometry was governed by one of the parameters in the equation. The solution has been written in the form of convolution (memory) integrals that relate the local values of the transported quantity and its flux. Such solutions are valid everywhere within the domain, including the domain boundary.

Furthermore, it has been shown that, in many special cases, the solution reduces to less general solutions that have been reported previously.

To validate the model, various solutions of the resulting integral equation have been numerically obtained. In the case of the planar geometry, all these solutions coincide with the solutions obtained in previous works. Furthermore, it has been shown that the solutions obtained in the case of the spherical geometry converge to the corresponding planar solutions as the radius of the surface curvature increases.

In addition, the solution given by (4.17) provides some important clues of how energy transport processes take place in general. Thus, for instance, it follows that a certain maximal speed of energy transport should exist such that no process of energy transport may occur with a speed larger than that maximum speed (the parameter $c$ and the term $\sqrt{1-u^{2} / c^{2}}$ in the solution). Furthermore, although allowed being very small, the time lag $\tau$ in the solution is finite. This time lag may be viewed as the "time quantum" of the process in question. Moreover, the solution contains the "space quantum" $\ell$ defined as $\ell=c \tau$.

Curiously enough, in the case of a nonzero value of the velocity $u$, the solution given by (4.17) becomes a mapping of the form $F_{n+1}=\mathfrak{J}\left(F_{n}\right)$, where $\mathfrak{J}$ denotes the integral operator in (4.17). Therefore, the solution of the generalized equation of energy transport allows chaotic or even biotic (self-organized) solutions [13]. This may become the topic of future studies.

\section{References}

[1] M. Abramowitz and I. A. Stegun, Handbook of Mathematical Functions, with Formulas, Graphs, and Mathematical Tables, Dover Publications, New York, 1965.

[2] V. V. Kulish, Fractional solutions for near-wall turbulence production, Fractional Differentiation and Its Applications, Bordeaux, 2004.

[3] __ Modeling of gas exchange in a single alveolus: mass transfer in an expanding sphere, 15th International Symposium on Transport Phenomena, Bangkok, 2004. 
[4] V. V. Kulish and J. L. Lage, Fractional-diffusion solutions for transient temperature and heat transfer, ASME J. Heat Transfer 122 (2000), no. 2, 372-376.

[5] Application of fractional calculus to fluid mechanics, ASME J. Fluid Engineering 124 (2002), no. 3, 803-806.

[6] V. V. Kulish, J. L. Lage, P. L. Komarov, and P. E. Raad, A Fractional-diffusion theory for calculating thermal properties of thin films from surface transient thermoreflectance measurements, ASME J. Heat Transfer 123 (2001), no. 6, 1133-1138.

[7] V. V. Kulish and V. B. Novozhilov, Relationship between the local temperature and local heat flux in a one-dimensional semi-infinite domain with the moving boundary, AIAA J. of Thermophysics \& Heat Transfer 17 (2003), no. 4, 538-540.

[8] - The relationship between the local temperature and the local heat flux within a onedimensional semi-infinite domain of heat wave propagation, Math. Probl. Eng. 2003 (2003), no. $4,173-179$.

[9] - On the integral equation for the neural response to an external stimulus, 5th International Conference on Mathematical Problems in Engineering \& Aerospace Sciences, Timisoara, 2004.

[10] V. V. Kulish and A. I. Sourin, Simulation and visualization of thermal wave propagation in picoscales, 10th International Symposium on Flow Visualization, Kyoto, 2002.

[11] Simulation and visualization of thermal wave propagation in sub-nano-scales: ultra-fast laser heating of solid materials, 7th Asian Symposium on Visualization, Singapore, 2003.

[12] K. B. Oldham and J. Spanier, The Fractional Calculus, Academic Press, New York, 1974.

[13] H. Sabelli and A. Abouzeid, Definition and empirical characterization of creative processes, Nonlinear Dynamics Psychol. Life Sci. 7 (2003), no. 1, 35-47.

Vladimir V. Kulish: School of Mechanical and Production Engineering, Nanyang Technological University, Singapore 639798

E-mail address: mvvkulish@ntu.edu.sg 


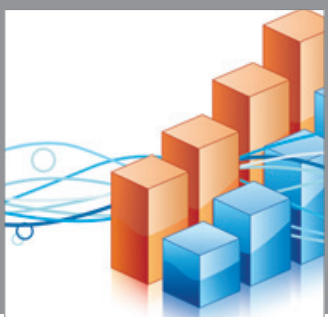

Advances in

Operations Research

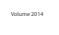

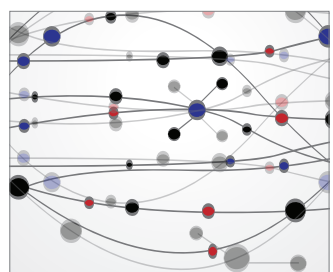

\section{The Scientific} World Journal
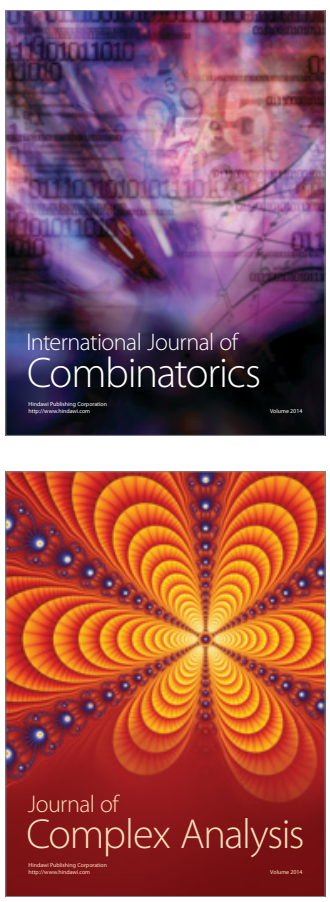

International Journal of

Mathematics and

Mathematical

Sciences
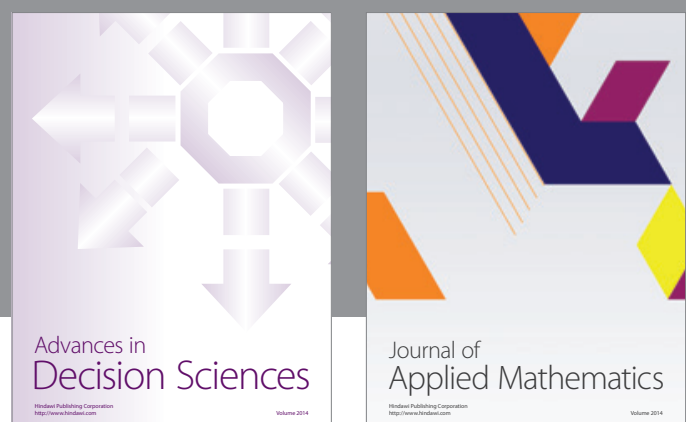

Journal of

Applied Mathematics
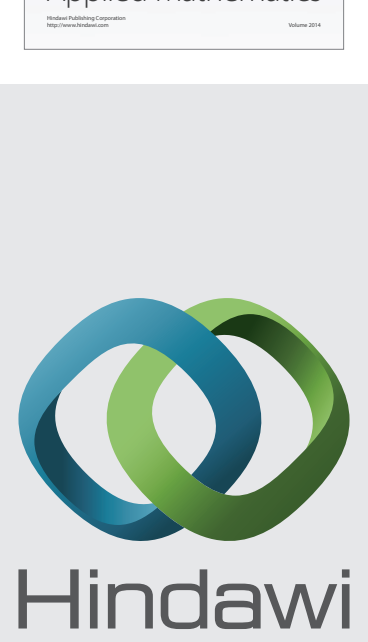

Submit your manuscripts at http://www.hindawi.com
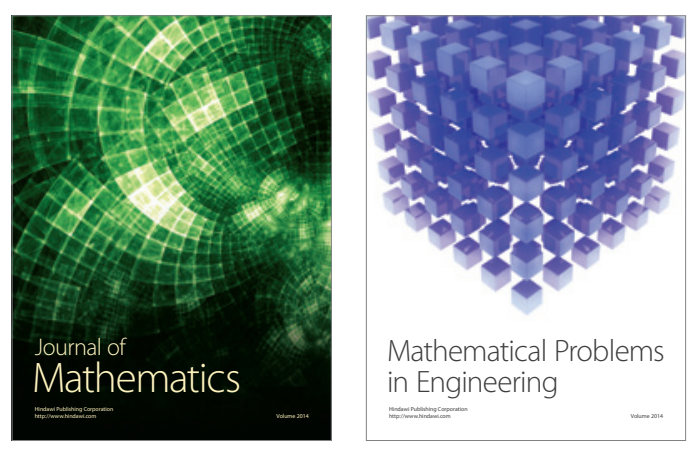

Mathematical Problems in Engineering
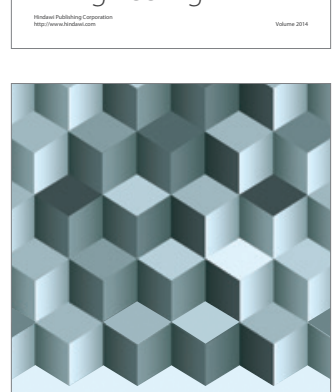

Journal of

Function Spaces
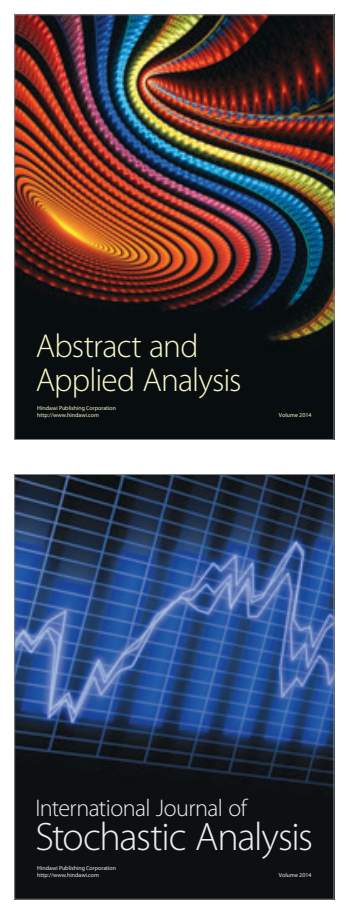

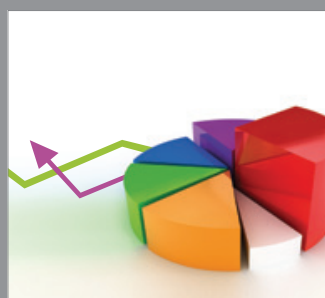

ournal of

Probability and Statistics

Promensencen
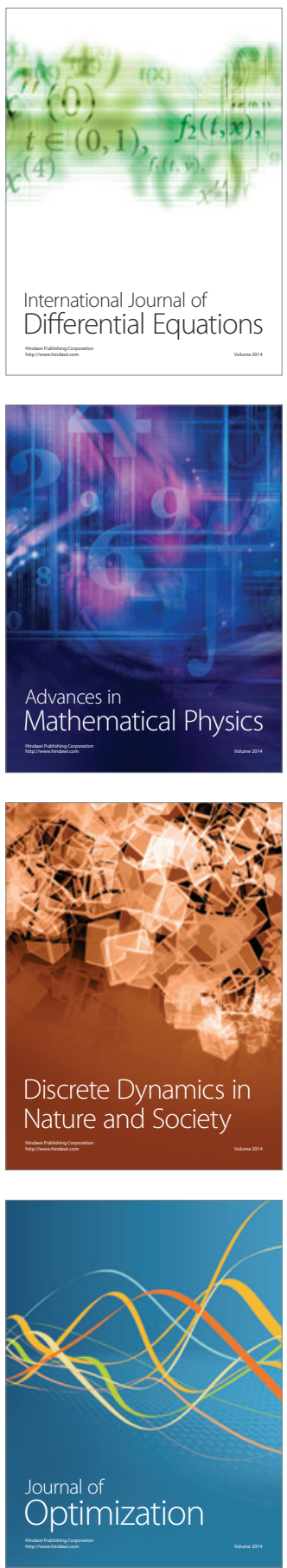